\title{
Multi-Channel Tri-gate GaN Power Schottky Diodes with Low ON-Resistance
}

\author{
Jun Ma, George Kampitsis, Member, IEEE, Peng Xiang, Kai Cheng and Elison Matioli, Member, \\ IEEE
}

\begin{abstract}
In this work we demonstrate high-performance lateral GaN power Schottky barrier diodes (SBDs) based on a novel multi-channel tri-gate architecture. A significant reduction in $\mathrm{ON}$ resistance $(R O N)$ of $50 \%$, down to $7.2 \pm 0.4 \Omega \cdot \mathbf{m m}$, along with a much smaller forward voltage $\left(V_{F}\right)$ of $1.57 \pm 0.06 \mathrm{~V}$, were achieved with multiple 2DEG channels (multi-channels) formed by periodic AlGaN/GaN heterostructures. We used a tri-anode structure to form Schottky contact to the multi-channels through the fin sidewalls, leading to a small turn-ON voltage $\left(V_{O N}\right)$ of $0.67 \pm 0.04$ $V$. To simultaneously control the multi-channels and effectively spread the electric field in OFF state, a tri-gate structure was integrated in the anode, resulting in an ultra-low leakage current $\left(I_{R}\right)$ of $\sim 1 \mathrm{nA} / \mathrm{mm}$ at $-600 \mathrm{~V}$ and a high breakdown voltage $\left(V_{\mathrm{BR}}\right)$ of $-900 \mathrm{~V}$ at $1 \mu \mathrm{A} / \mathrm{mm}$ with grounded substrate. In addition, the devices presented promising switching performance, due to the small product of $R_{O N}$ and reverse charge $(Q)$, thanks to the optimized tri-gate geometry, and the high effective mobility $\left(\mu_{\mathrm{e}}\right)$ of $2063 \pm 123 \mathrm{~cm}^{2} \cdot \mathrm{V}^{-1} \mathrm{~s}^{-1}$ despite the small fin width $(w)$ of $50 \mathrm{~nm}$. Our approach combines in a unique way the excellent electrostatic control of the tri-gate structure with the high conductivity of multi-channels, offering a promising platform for future advances in $\mathrm{GaN}$ power devices.
\end{abstract}

Index Terms-GaN, SBD, multi-channel, tri-gate, tri-anode, breakdown, leakage current.

\section{INTRODUCTION}

$\mathrm{G}$ aN-on-Si SBDs are promising as power rectifiers [1]-[10], offering high performance at a competitive cost, and can be integrated with $\mathrm{GaN}$ transistors to form advanced integrated power devices [11]-[14] and circuits [15]-[18]. However, a major challenge is to achieve high reverse-blocking capabilities along with low forward-conduction losses, which is an intrinsic trade-off that limits many other types of power devices.

In this work we present novel multi-channel tri-gate SBDs to address this challenge (Fig. 1). We employed periodic AlGaN/GaN heterostructures with multiple 2DEG channels [19]-[22] to reduce the $R_{\mathrm{ON}}$ and $V_{\mathrm{F}}$. 3D tri-anode and tri-gate electrodes were implemented to contact and control the multichannels, resulting in small $V_{\mathrm{ON}}$, low $I_{\mathrm{R}}$, and high $V_{\mathrm{BR}}$. This unique design significantly enhanced the device performance, leading to state-of-the-art lateral GaN-on-Si power SBDs, and unveiled a novel platform to drastically improve the efficiency, increase the current rating, and reduce the size of GaN-based power devices.

This work was supported in part by the European Research Council under the European Union's H2020 program/ERC grant Agreement 679425 and in part by the Swiss National Science Foundation under Assistant Professor (AP) Energy Grant PYAPP2_166901.
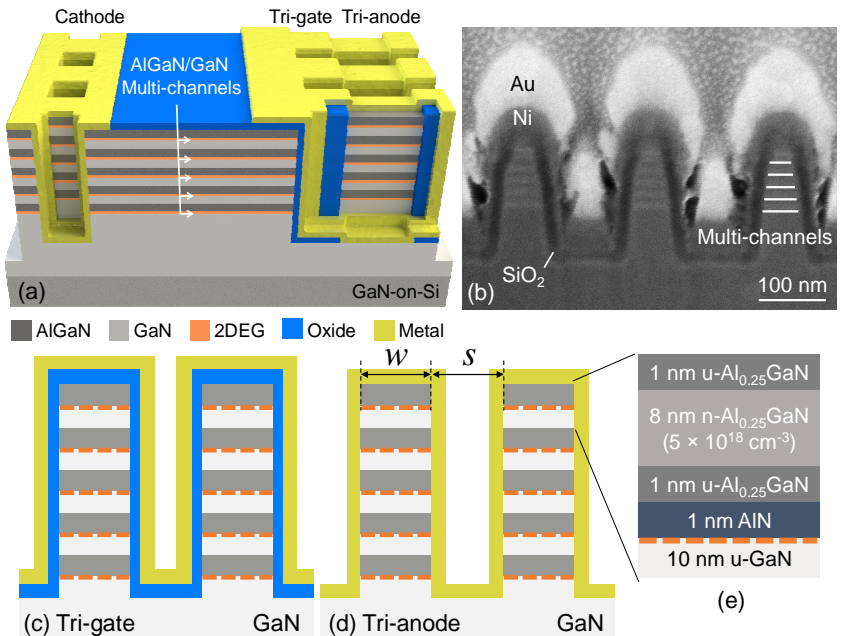

Fig. 1. (a) Schematic of the multi-channel tri-gate SBD. (b) Cross-sectional SEM image of the multi-channel tri-gate region, tilted by $52^{\circ}$. Cross-sectional schematics of the (c) tri-gate and (d) tri-gate regions. (e) Schematic of the heterostructure composing each channel in the multi-channel structure.

\section{DEVICE DESIGN AND FABRICATION}

The multi-channel AlGaN/GaN heterostructure in this work consisted of 5 parallel 2DEG channels, each composed of 10 $\mathrm{nm}$ AlGaN barrier, $1 \mathrm{~nm}$ AlN spacer and $10 \mathrm{~nm}$ GaN channel layers, in which the barrier was selectively doped with $\mathrm{Si}$ at $5 \times$ $10^{18} \mathrm{~cm}^{-3}$. The heterostructure was grown on Si substrate with a $4.3 \mu \mathrm{m}$-thick buffer layer, exhibiting a small sheet resistance $\left(R_{\mathrm{S}}\right)$ of $230 \Omega / \mathrm{sq}$, along with large carrier concentration $\left(N_{\mathrm{s}}\right)$ of $1.5 \times 10^{13} \mathrm{~cm}^{-2}$ and hall electron mobility of $1820 \mathrm{~cm}^{2} \cdot \mathrm{V}^{-1} \mathrm{~s}^{-1}$. The anode region was selectively patterned into fins with designed height of $200 \mathrm{~nm}$ and width $(w)$ of $50 \mathrm{~nm}$, using $\mathrm{Ar} / \mathrm{Cl}_{2}$-based inductively coupled plasma etching, on which the tri-gate (Fig. 1(b)) and tri-anode were formed over the parallel channels. The lengths of the cathode-to-anode $\left(L_{\mathrm{AC}}\right)$, tri-gate and tri-anode regions were $15 \mu \mathrm{m}, 1.2 \mu \mathrm{m}$ and $4 \mu \mathrm{m}$, respectively. The tri-anode contacts the multi-channels through the fin sidewalls, resulting in a small $V_{\mathrm{ON}}$. The tri-gate/tri-anode regions shield the sidewall Schottky junction from the high electric fields under large reverse biases to reduce $I_{\mathrm{R}}$ [1],[23]. The tri-gate region serves as a field plate, and converts the planar region of the anode into a second field plate, improving the $V_{\mathrm{BR}}$ [24]. The ohmic region in the cathode was patterned

J. Ma, G. Kampitsis, and E. Matioli are with École polytechnique fédérale de Lausanne (EPFL), CH-1015 Lausanne, Switzerland. (e-mail: jun.ma@epfl.ch; elison.matioli@epfl.ch).P.Xiang and K. Cheng are with Enkris Semiconductor Inc., Suzhou 215123, P. R. China. 

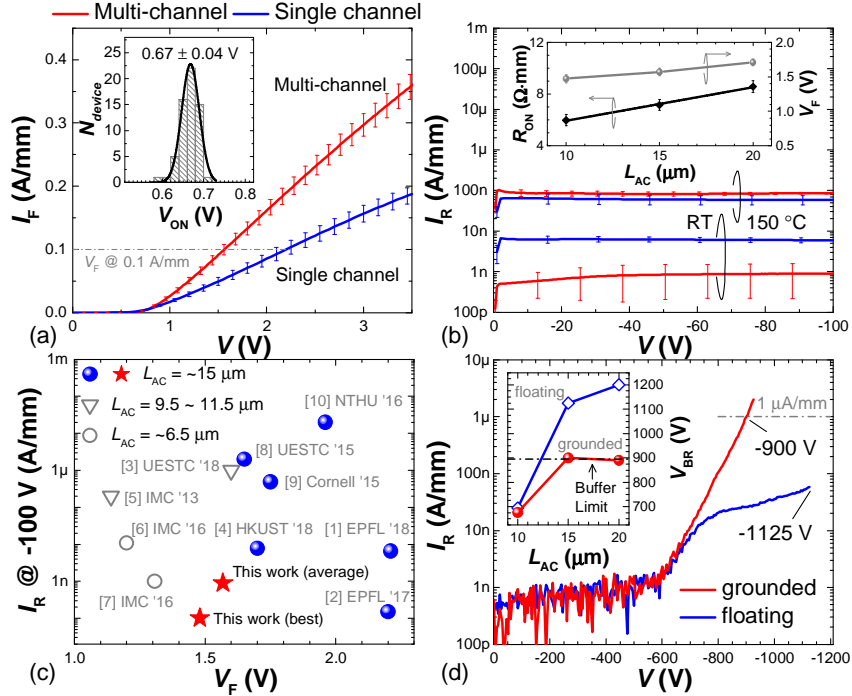

(d)

$V(\mathrm{~V})$

Fig. 2. (a) Forward and (b) reverse characteristics of the multi-channel tri-gate SBDs. (c) Comparison of $I_{\mathrm{R}}$ and $V_{\mathrm{F}}$ of the GaN-on-Si SBDs in this work and literature. (d) Breakdown characteristics of the multi-channel tri-gate SBDs. The inset in (a) shows the distribution of the $V_{\mathrm{ON}}$. The inset in (b) shows the dependence of $R_{\mathrm{ON}}$ and $V_{\mathrm{F}}$ upon $L_{\mathrm{AC}}$ in multi-channel tri-gate SBDs. The inset in (d) shows the dependence of $V_{\mathrm{BR}}$ upon $L_{\mathrm{AC}}$ in multi-channel tri-gate SBDs.

- Lateral GaN-on-Si SBDs ( $V_{\text {ON }}, I_{\mathrm{B}}$ at $-650 \mathrm{~V}$ (if applicable))

- Vertical GaN-on-GaN SBDs $\Delta$ Cree SBDs (ON/OFF ratio at -600 V (if applicable)) $\star$ This work: lateral GaN-on-Si multi-channel tri-gate SBDs

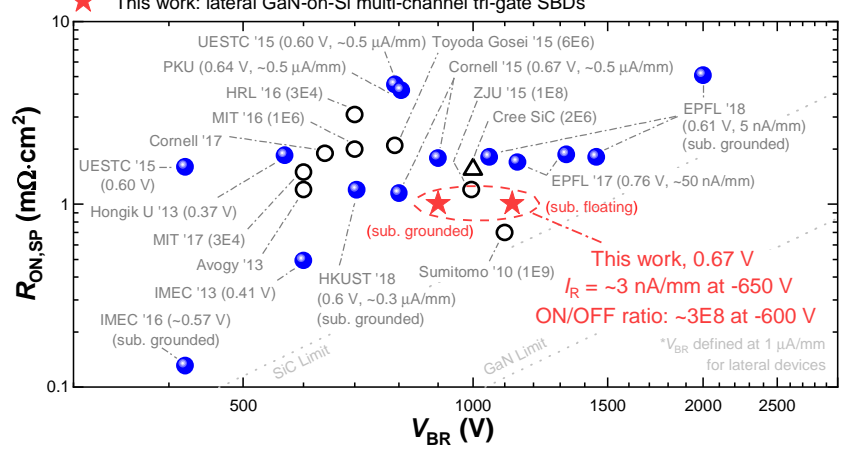

Fig. 3. Specific on-resistance $\left(R_{\mathrm{ON}, \mathrm{SP}}\right)$ versus $V_{\mathrm{BR}}$ benchmark of the multichannel tri-gate SBDs against state-of-the-art lateral GaN SBDs. For fair comparison, the $V_{\mathrm{BR}}$ for all lateral devices was re-calculated based on the reported data following the definition of $V_{\mathrm{BR}}$ at $I_{\mathrm{R}}=1 \mu \mathrm{A} / \mathrm{mm}$, and devices with unspecified $R_{\mathrm{ON}}$ or $I_{\mathrm{R}}$ were not included. Unfortunately the references could not be included here due to the limit in length, however they can be found in Ref. [1] for lateral devices and Refs. [25] for vertical devices.

with $w$ and $s$ of $500 \mathrm{~nm}$, to contact all parallel channels at the fin sidewalls. In the tri-gate region, $25 \mathrm{~nm} \mathrm{SiO} 2$ was deposited by atomic layer deposition as the oxide, and $\mathrm{Ni} / \mathrm{Au}$ were used as the anode metals. Optimal single-channel SBDs with similar device architecture and dimensions in Ref. [1] were used as reference, which have high mobility of $2000 \mathrm{~cm}^{2} \cdot \mathrm{V}^{-1} \mathrm{~s}^{-1}$ and $N_{\mathrm{s}}$ of $1 \times 10^{13} \mathrm{~cm}^{-2}$. All current values in this work were normalized by the width of the device footprint $(60 \mu \mathrm{m})$, and the error bars were determined from at least 12 devices of each type.

\section{RESULTS AND DISCUSSION}

The multi-channel tri-gate SBDs exhibited considerably enhanced performance over counterpart single-channel devices. The $R_{\mathrm{ON}}$ was reduced by $\sim 50 \%$ to $7.2 \pm 0.4 \Omega \cdot \mathrm{mm}$, the forward current $\left(I_{\mathrm{F}}\right)$ was increased by $\sim 100 \%$ at $3.5 \mathrm{~V}$, and $V_{\mathrm{F}}$ was decreased from $2.21 \pm 0.14 \mathrm{~V}$ to $1.57 \pm 0.06 \mathrm{~V}($ at $0.1 \mathrm{~A} / \mathrm{mm})$
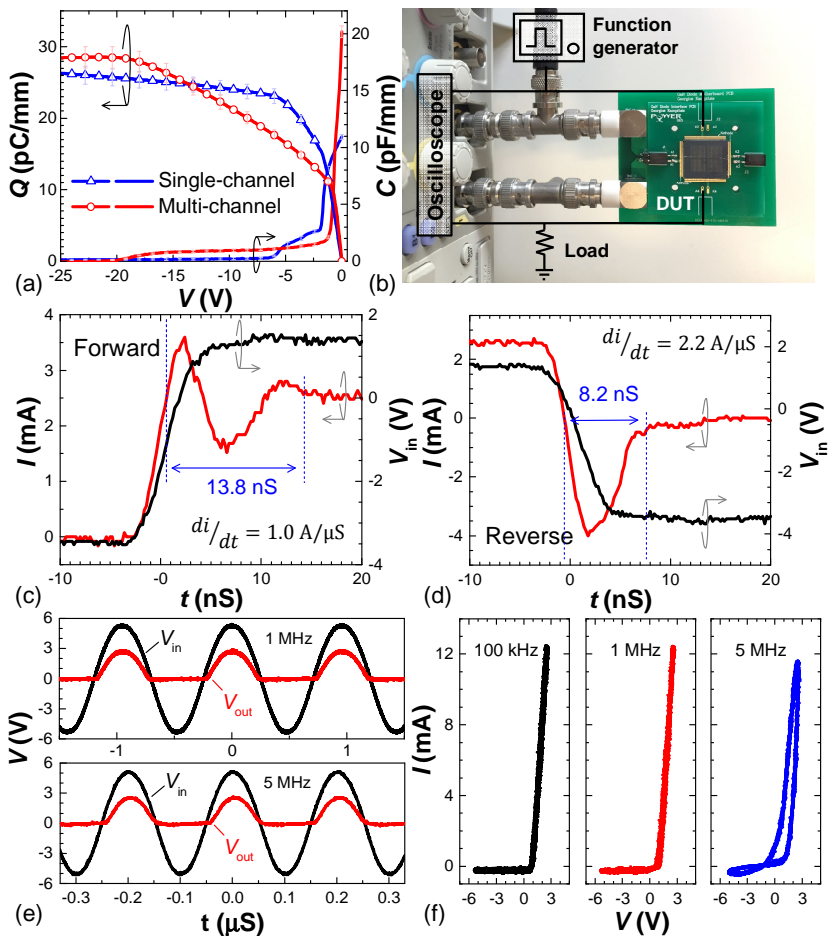

Fig. 4. (a) Capacitance $(C)$ and $O$ in multi-channel and single-channel SBDs. (b) Setup for measuring the switching characteristics. The device was bonded onto a printed circuit board (PCB) and connected directly to the oscilloscope to minimize parasitic elements. (c) Forward- and (d) reverse-recovery characteristics of the multi-channel tri-gate SBDs. The devices were switched between $1.5 \mathrm{~V}$ to $-3.5 \mathrm{~V}$ at $1 \mathrm{MHz}$ (limited by the oscilloscope) using the internal oscilloscope function generator and a $50 \Omega$ resistor as the load. (e) Input $\left(V_{\text {in }}\right)$ and output $\left(V_{\text {out }}\right)$ waveforms and (f) Lissajous $I-V$ plots showing the rectification characteristics of the multi-channel tri-gate SBDs at different frequencies, using an external function generator and a $220 \Omega$ resistor. The small phase shift in (e) between $V_{\text {in }}$ and $V_{\text {out }}$ was likely caused by parasitic elements in the circuit.

in multi-channel tri-gate SBDs, while exhibiting a similar $V_{\mathrm{ON}}$ of $0.67 \pm 0.04 \mathrm{~V}$ at $1 \mathrm{~mA} / \mathrm{mm}$ (Fig. 2(a)). In these results, the spacing $(s)$ between the fins was $100 \mathrm{~nm}$, yielding a filling factor $(F F)$ of $33 \%$. The $V_{\mathrm{F}}$ and $R_{\mathrm{ON}}$ in multi-channel tri-gate SBDs changed very slightly with the $L_{\mathrm{AC}}$ (the inset in Fig. 2(b)), which is beneficial for high-voltage lateral devices (requiring a large $L_{\mathrm{AC}}$ ). In addition to the enhanced $\mathrm{ON}$-state conductivity, the multi-channel tri-gate SBDs exhibited a $6 \mathrm{x}$-smaller average $I_{\mathrm{R}}$ of $0.89 \mathrm{nA} / \mathrm{mm}$ at $-100 \mathrm{~V}$, which increased to only $86 \pm 4$ $\mathrm{nA} / \mathrm{mm}$ at $150{ }^{\circ} \mathrm{C}$ (Fig. 2(b)), revealing the excellent electrostatic control of the tri-gate over the multi-channels even at high temperature. The small $V_{\mathrm{F}}$ and $I_{\mathrm{R}}$ in this work yields better device performance, in both ON and OFF states, than in other lateral GaN SBDs with similar dimensions (Fig. 2(c)).

The multi-channel tri-gate SBDs also presented excellent voltage-blocking performance (Fig. 2(d)). The $V_{\mathrm{BR}}$ at $1 \mu \mathrm{A} / \mathrm{mm}$ was as high as $900 \mathrm{~V}$ with grounded substrate, limited by the $4.3 \mu \mathrm{m}$ buffer layer (inset in Fig. 2(d)), and the $I_{\mathrm{R}}$ at $-650 \mathrm{~V}$ was only $\sim 3 \mathrm{nA} / \mathrm{mm}$, which is much smaller than in other reports (Fig. 2(e)). Such high voltage-blocking performance indicate the potential of these devices for $650 \mathrm{~V}$ applications, providing an small $I_{\mathrm{R}}$ at the rated voltage and a safety margin of $\sim 50 \%$ from the rated voltage to the hard breakdown. The multichannel tri-gate SBDs presented superb performance and excellent high-power figure-of-merit up to $1.25 \mathrm{GW} / \mathrm{cm}^{2}$ (Fig. 


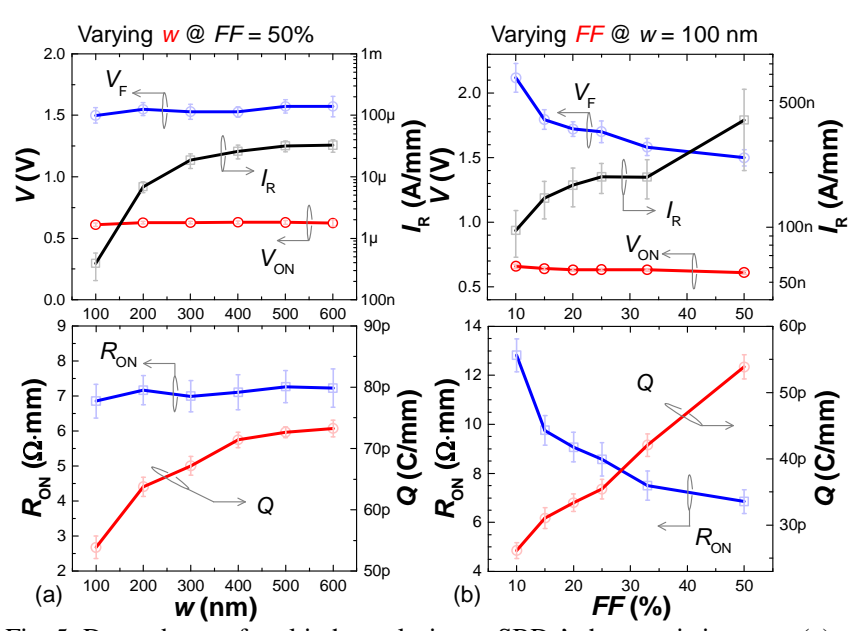

Fig. 5. Dependence of multi-channel tri-gate SBDs' characteristics upon (a) $w$ and (b) $F F$ in tri-gate and tri-anode regions.

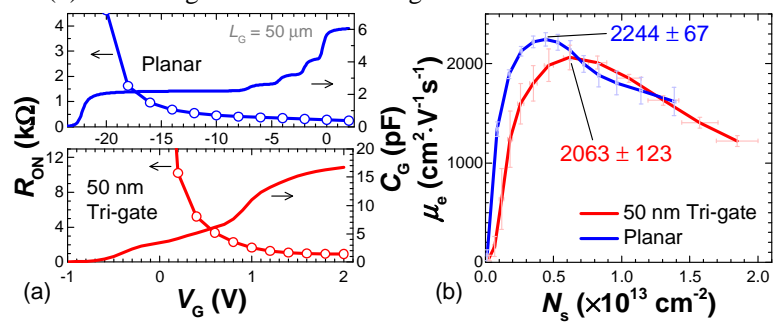

Fig. 6. (a) Average $R_{\mathrm{ON}}$ and $C_{\mathrm{G}}$ in multi-channel MOSHEMTs with planar-gates and $50 \mathrm{~nm}$-wide tri-gates. The $L_{\mathrm{G}}$ was $50 \mu \mathrm{m}$ for both devices, and the gate-tosource and gate-to-drain distances were both $1.5 \mu \mathrm{m}$. $R_{\mathrm{ON}}$ was measured using drain voltages below $0.1 \mathrm{~V}$, and $C_{\mathrm{G}}$ was measured at $1 \mathrm{MHz}$. (b) $\mu_{\mathrm{e}}$ versus $N_{\mathrm{s}}$ in the two devices. The $N_{\mathrm{s}}$ was normalized by the top surface area of the fins, which did not impact the determination of $\mu_{\mathrm{e}}$

3), comparable to state-of-the-art GaN-on-GaN vertical SBDs [25],[26] as well as GaN-on-Si power transistors [27].

The switching performance in multi-channel tri-gate SBDs is very promising. Despite the $\sim 50 \%$ reduction in $R_{\mathrm{ON}}$, the charge $(Q)$ in multi-channel SBDs increased by only $8.4 \%$ to $28.5 \pm$ $1.5 \mathrm{pC} / \mathrm{mm}$ (Fig. 4(a)), which is smaller than in conventional fast-switching GaN-on-Si power SBDs [9],[28], resulting in a much smaller $R_{\mathrm{ON}} \cdot Q$ product and thus offering great potential for efficient power rectification at high frequencies. The switching performance of the multi-channel tri-gate SBDs was investigated using a rectification circuit [29] with minimized parasitic elements through an optimized PCB layout and short interconnections (Fig. 4(b)). At a frequency of $1 \mathrm{MHz}$, the forward-recovery time of the multi-channel tri-gate SBDs was as small as $13.8 \mathrm{~ns}$ (Fig. 4(c)), along with a very short reverserecovery time of $8.2 \mathrm{~ns}$ (Fig. 4(d)), which did not change from $10 \mathrm{kHz}$ to $10 \mathrm{MHz}$. Figures 4(e) and (f) show the $V_{\text {in }}$ and $V_{\text {out }}$ waveforms as well as Lissajous plots of the $I-V$ characteristics of the multi-channel tri-gate SBDs, respectively, which indicate an effective rectification by these devices up to $5 \mathrm{MHz}$ (at this frequency a phase shift between the $V$ and $I$ was observed).

The high performance of multi-channel tri-gate SBDs depends significantly on the tri-gate/tri-anode geometry to balance the ON-state, OFF-state and switching characteristics. As shown in Fig. 5(a), a smaller $w$ diminishes $I_{\mathrm{R}}$ and $Q$, but does not significantly degrade $R_{\mathrm{ON}}, V_{\mathrm{ON}}$ and $V_{\mathrm{F}}$, thus resulting in a reduced $R_{\mathrm{ON}} \cdot Q$ product. The reduction in $I_{\mathrm{R}}$ is due to the smaller pinch-off voltages in tri-gate/tri-anode regions with decreasing $w$, which lowers the electric field at the Schottky junction and exponentially reduces the $I_{\mathrm{R}}$ [23]. The smaller $Q$ is due to the reduced $N_{\mathrm{s}}$ [30] and smaller threshold voltages in the trigate/tri-anode regions as $w$ decreases [1]. Therefore, to have an $I_{\mathrm{R}}$ below $0.1 \mu \mathrm{A} / \mathrm{mm}$ for efficient power devices, $w$ needs to be smaller than $100 \mathrm{~nm}$ for the heterostructure used in this work (Fig. 4(a)). FF is another important variable (Fig. 5(b)). A decrease in $F F$ yields a reduction in $I_{\mathrm{R}}$ and $Q$, but an increase in $V_{\mathrm{F}}, V_{\mathrm{ON}}$ and $R_{\mathrm{ON}}$, thus leading to an optimal $F F$ of $33 \%$ for both a small $R_{\mathrm{ON}}$ and $R_{\mathrm{ON}} \cdot Q$ product.

Another reason for the excellent performance in the multichannel tri-gate SBDs is the high $\mu_{\mathrm{e}}$ in their tri-gate/tri-anode regions. To extract the $\mu_{\mathrm{e}}$, we fabricated multi-channel MOSHEMTs with $50 \mu \mathrm{m}$-long planar-gate and tri-gate structures. We measured the $R_{\mathrm{ON}}$, and integrated the measured gate capacitance $\left(C_{\mathrm{G}}\right)$ at different gate voltages $\left(V_{\mathrm{G}}\right)($ Fig. 6(a)) to extract the gate charge $\left(Q_{\mathrm{G}}\right)$. The $\mu_{\mathrm{e}}$ was obtained using $R_{\mathrm{ON}}$ $\approx L_{\mathrm{G}} /\left(W_{\mathrm{G}} \cdot N_{\mathrm{s}} \cdot q \cdot \mu_{\mathrm{e}}\right)=L_{\mathrm{G}}{ }^{2} /\left(Q_{\mathrm{G}} \cdot \mu_{\mathrm{e}}\right)$, in which $L_{\mathrm{G}}$ and $W_{\mathrm{G}}$ are the gate length and width, respectively, $q$ is the elementary charge, and $N_{\mathrm{s}}$ is the 2DEG concentration in gate region. This method eliminates possible errors in $W_{\mathrm{G}}$ from variation in lithography or normalization. The $\mu_{\mathrm{e}}$ in the tri-gate region was as high as $2063 \pm 123 \mathrm{~cm}^{2} \cdot \mathrm{V}^{-1} \mathrm{~s}^{-1}$, which is comparable to that in planargate multi-channel devices $\left(2244 \pm 67 \mathrm{~cm}^{2} \cdot \mathrm{V}^{-1} \mathrm{~s}^{-1}\right)$ (Fig. 6(b)), revealing a negligible reduction in $\mu_{\mathrm{e}}$ despite the small $w$ of 50 $\mathrm{nm}$, which is much higher than in conventional single-channel $\mathrm{AlGaN} / \mathrm{GaN}$ fin structures [31]-[33].

The future prospects for the multi-channel tri-gate technology are highly promising. Firstly, it can lead to novel lateral power devices with much smaller $R_{\mathrm{ON}}$ and much higher current ratings for a given device area, thanks to its ultra-low $R_{\mathrm{s}}$, down to $37 \Omega / \mathrm{sq}$ [19], combined with the superior voltageblocking capabilities of tri-gates and slanted tri-gates [1],[27]. Secondly, this approach can be easily extended for high-voltage normally-on/off multi-channel tri-gate GaN transistors [34], nanoscale in-plane-gate transistors, and many other devices and applications, yielding a promising platform for future efficient electronic devices. To unleash the full potential of multichannel tri-gate technology, an intensive and coupled material research and device engineering are required, which opens tremendous opportunities for future studies in novel epitaxy structures and device designs.

\section{CONCLUSION}

In this work we demonstrated novel multi-channel tri-gate GaN-on-Si power SBDs. The devices presented small $R_{\mathrm{ON}}$ and $V_{\mathrm{F}}$, due to the multiple 2DEG channels, and large $V_{\mathrm{BR}}$ and ultralow $I_{\mathrm{R}}$, thanks to the tri-gate, demonstrating state-of-the-art performance for $600 \mathrm{~V} / 650 \mathrm{~V}$ ratings.

\section{REFERENCES}

[1] J. Ma and E. Matioli, " 2 kV slanted tri-gate GaN-on-Si Schottky barrier diodes with ultra-low leakage current," Appl. Phys. Lett., vol. 112, pp. 052101-1-052101-4, Jan. 2018. doi: 10.1063/1.5012866.

[2] J. Ma and E. Matioli, "High-voltage and low-leakage AlGaN/GaN trianode Schottky diodes with integrated tri-gate transistors," IEEE Electron Device Lett., vol. 38, pp. 83-86, Jan. $2017 . \quad$ doi: 10.1109/LED.2016.2632044.

[3] A. Zhang, Q. Zhou, C. Yang, Y. Shi, C. Dong, T. Liu, Y. Shi, W. Chen, Z. Li, and B. Zhang, "Novel AlGaN/GaN SBDs with nanoscale multichannel for gradient 2DEG modulation," 2018 IEEE 30th International 
Symposium on Power Semiconductor Devices and ICs (ISPSD), Chicago, IL, 2018, pp. 204-207. doi: 10.1109/ISPSD.2018.8393638.

[4] J. Lei, J. Wei, G. Tang, Z. Zhang, Q. Qian, Z. Zheng, M. Hua, and K. J. Chen, "650-V Double-channel lateral Schottky barrier diode with dualrecess gated anode," IEEE Electron Device Lett., vol. 39, no. 2, pp. 260 263, Feb. 2018. doi: 10.1109/LED.2017.2783908.

[5] S. Lenci, B. D. Jaeger, L. Carbonell, J. Hu, G. Mannaert, D. Wellekens, S. You, B. Bakeroot, and S. Decoutere, "Au-free AlGaN/GaN power diode on 8-in Si substrate with gated edge termination," IEEE Electron Device Lett., vol. 34, pp. 1035-1037, Jul. 2013. doi: 10.1109/LED.2013.2267933.

[6] J. Hu, S. Stoffels, S. Lenci, B. Bakeroot, B. D. Jaeger, M. V. Hove, N. Ronchi, R. Venegas, H. Liang, M. Zhao, G. Groeseneken, and S Decoutere, "Performance optimizaiton of Au-free lateral AlGaN/GaN Schottky barrier diode with gated edge termination on 200-mm silicon substrate," IEEE Trans. Electron Devices, vol. 63, pp. 997-1004, Jan. 2016. doi: 10.1109/TED.2016.2515566.

[7] J. Hu, S. Stoffels, S. Lenci, B. D. Jaeger, N. Ronchi, A. N. Tallarico, D. Wellekens, S. You, B. Bakeroot, G. Groeseneken, and S. Decoutere, "Statistical analysis of the impact of anode recess on electrical characteristics of AlGaN/GaN Schottky diodes with gated edge termination," IEEE Trans. Electron Devices, vol. 63, pp. 3451-3458, Jul. 2016. doi: 10.1109/TED.2016.2587103.

[8] Q. Zhou, Y. Jin, Y. Shi, J. Mou, X. Bao, B. Chen, and B. Zhang, "High reverse blocking and low onset voltage $\mathrm{AlGaN} / \mathrm{GaN}$-on-Si lateral power diode with MIS-gated hybrid anode," IEEE Electron Device Lett., vol. 36, pp. 660-662, May 2015. doi: 10.1109/LED.2015.2432171.

[9] M. Zhu, B. Song, M. Qi, Z. Hu, K. Nomoto, X. Yan, Y. Cao, W. Johnson, E. Kohn, D. Jena, H. G. Xing, "1.9-kV AlGaN/GaN lateral Schottky barrier diodes on silicon," IEEE Electron Device Lett., vol. 36, pp. 375377, Feb. 2015. doi: 10.1109/LED.2015.2404309.

[10] C. -W. Tsou, K. -P. Wei, Y. -W. Lian, and S. S. H. Hsu, "2.07-kV AlGaN/GaN Schottky barrier diodes on silicon with high Baliga's Figureof-Merit," IEEE Electron Device Lett., vol. 37, pp. 70-73, Jan. 2016. doi: 10.1109/LED.2015.2499267.

[11] J. Ma, M. Zhu and E. Matioli, "900 V Reverse-blocking GaN-on-Si MOSHEMTs with a hybrid tri-Anode Schottky drain," IEEE Electron Device Lett., vol. 38, no. 12, pp. 1704-1707, Dec. 2017. doi: 10.1109/LED.2017.2761911.

[12] T. Wang, J. Ma and E. Matioli, "1100 V AlGaN/GaN MOSHEMTs with integrated tri-anode freewheeling diodes," IEEE Electron Device Lett. vol. 39, no. 7, pp. 1038-1041, Jul. 2018. doi: 10.1109/LED.2018.2842031.

[13] J. Lei, J. Wei, G. Tang, Z. Zhang, Q. Qian, Z. Zheng, M. Hua, and K. J. Chen, "Reverse-blocking normally-off GaN double-channel MOSHEMT with low reverse leakage current and low ON-state resistance," IEEE Electron Device Lett., vol. 39, no. 7, pp. 1003-1006, July 2018. doi: 10.1109/LED.2018.2832180.

[14] J. Lei. J. Wei, G. Tang, Q. Qian, M. Hua, Z. Zhang, Z. Zheng, and K. J. Chen, "An interdigitated GaN MIS-HEMT/SBD normally-off power switching device with low ON-resistance and low reverse conduction loss," 2017 IEEE International Electron Devices Meeting (IEDM), San Francisco, CA, 2017, pp. 25.2.1-25.2.4. doi: 10.1109/IEDM.2017.8268456.

[15] S. Ujita, Y. Kinoshita, H. Umeda, T. Morita, K. Kaibara, S. Tamura, M. Ishida, and T. Ueda, "A fully integrated GaN-based power IC including gate drivers for high-efficiency DC-DC Converters," 2016 IEEE Symposium on VLSI Circuits (VLSI-Circuits), Honolulu, HI, 2016, pp. 12. doi: 10.1109/VLSIC.2016.7573496.

[16] D. Maksimović, Y. Zhang and M. Rodríguez, "Monolithic very high frequency GaN switched-mode power converters," 2015 IEEE Custom Integrated Circuits Conference (CICC), San Jose, CA, 2015, pp. 1-4. doi: 10.1109/CICC.2015.7338386.

[17] W. Chen, K. Y. Wong and K. J. Chen, "Single-chip boost converter using monolithically integrated $\mathrm{AlGaN} / \mathrm{GaN}$ lateral field-effect rectifier and normally off HEMT," IEEE Electron Device Lett., vol. 30, no. 5, pp. 430432, May 2009. doi: 10.1109/LED.2009.2015897.

[18] R. Reiner, P. Waltereit, B. Weiss, M. Wespel, M. Wespel, M. Mikulla, R. Quay, and O. Ambacher, "Monolithic GaN-on-Si half-bridge circuit with integrated freewheeling diodes," 2016 International Exhibition and Conference for Power Electronics, Nuremberg, Germany, 2016, pp. 1-7.

[19] Y. Cao, K. Wang, G. Li, T. Losel, H. Xing, and D. Jena, "MBE growth of high conductivity single and multiple AlN/GaN heterojunctions," $J$ Cryst. Growth, vol. 323, pp. 529-533, May 2011. doi: 10.1016/j.jcrysgro.2010.12.047.
[20] S. Heikman, S. Keller, D. S. Green, S. P. DenBaars, and U. K. Mishra, "High conductivity modulation doped AlGaN/GaN multiple channel heterostructures," J. Appl. Phys., vol. 94, no. 8, pp. 5321-5325, Oct. 2003. doi: 10.1063/1.1610244.

[21] Y. Zhang, Z. Wang, S. Xu, D. Chen, W. Bao, J. Zhang, J. Zhang, and Y. Hao, "Studies on the InAlN/InGaN/InAlN/InGaN double channel heterostructures with low sheet resistance," Appl. Phys. Lett., vol. 111, pp. 222107-1-222107-5, Dec. 2017. doi: 10.1063/1.4994656.

[22] H. Ishida, D. Shibata, H. Matsuo, M. Yanagihara, Y. Uemoto, T. Ueda, T. Tanaka, and D. Ueda, "GaN-based natural super junction diodes with multi-channel structures," 2008 IEEE International Electron Devices Meeting (IEDM), San Francisco, CA, 2008, pp. 1-4. doi: 10.1109/IEDM.2008.4796636.

[23] J. Ma, D. C. Zanuz and E. Matioli, "Field plate design for low leakage current in lateral GaN power Schottky diodes: role of the pinch-off voltage," IEEE Electron Device Lett., vol. 38, pp. 1298-1301, Sept. 2017. doi: 10.1109/LED.2017.2734644.

[24] J. Ma and E. Matioli, "High performance tri-gate GaN power MOSHEMTs on Silicon Substrate," IEEE Electron Device Lett., vol. 38, no. 3, pp. 367-370, Mar. 2017. doi: 10.1109/LED.2017.2661755.

[25] S. Han, S. Yang and K. Sheng, "High-voltage and high- $I_{\mathrm{ON}} / I_{\mathrm{OFF}}$ vertical GaN-on-GaN Schottky barrier diode with nitridation-based Termination," IEEE Electron Device Lett., vol. 39, no. 4, pp. 572-575, Apr. 2018. doi: 10.1109/LED.2018.2808684.

[26] Y. Saitoh, K. Sumiyoshi, M. Okada, T. Horii, T. Miyazaki, H. Shiomi, M. Ueno, K. Katayama, M. Kiyama, and T. Nakamura, "Extremely low onresistance and high breakdown voltage observed in vertical GaN Schottky barrier diodes with high-mobility drift layers on lowdislocation-density GaN substrates," Appl. Phys. Express, vol. 3, no. 8, p. 081001, Jul. 2010. doi: 10.1143/APEX.3.081001

[27] J. Ma and E. Matioli, "Slanted tri-gates for high-voltage GaN power devices," IEEE Electron Device Lett., vol. 38, , pp. 1305-1308, Sept. 2017. doi: 10.1109/LED.2017.2731799.

[28] E. Bahat-Treidel, O. Hilt, A. Wentzel, J. Würfl, and G. Tränkle, "Fast GaN based Schottky diodes on $\mathrm{Si}(111)$ substrate with low onset voltage and strong reverse blocking," Physics Status Solidi C, vol. 10, pp. 849852, Feb. 2013. doi: 10.1002/pssc.201200569.

[29] Q. He, W. Mu, B. Fu, Z. Jia, S. Long, Z. Yu, Z. Yao, W. Wang, H. Dong, Y. Qin, G. Jian, Y. Zhang, H. Xue, H. Lv, Q. Liu, M. Tang, X. Tao, and M. Liu, "Schottky Barrier Rectifier Based on (100) $\beta-\mathrm{Ga}_{2} \mathrm{O}_{3}$ and its DC and AC Characteristics," IEEE Electron Device Lett., vol. 39, no. 4, pp. 556-559, Apr. 2018. doi: 10.1109/LED.2018.2810858.

[30] J. Ma and E. Matioli, "Improved electrical and thermal performances in nanostructured GaN devices," 2016 International Conference on IC Design and Technology (ICICDT), Ho Chi Minh City, 2016, pp. 1-4. doi: 10.1109/ICICDT.2016.7542061.

[31] K. -S. Im, H. -S. Kang, D. -K. Kim, S. Vodapally, Y. Park, J. -H. Lee, Y. -T. Kim, S. Cristoloveanu, and J. -H. Lee, "Temperature-dependent characteristics of AlGaN/GaN FinFETs with sidewall MOS channel," Solid State Electron., vol. 120, pp. 47-51, Mar. 2013. doi: 10.1016/j.sse.2016.03.007.

[32] S. Joglekar, M. Azize, E. J. Jones, D. Piedra, S. Gradečak, and T. Palacios, "Impact of $\mathrm{Al}_{2} \mathrm{O}_{3}$ passivation on $\mathrm{AlGaN} / \mathrm{GaN}$ nanoribbon high-electronmobility transistors," IEEE Trans. Electron Devices, vol. 63, no. 1, pp. 318-325, Dec. 2016. doi: 10.1109/TED.2015.2500159.

[33] K. -S. Im, C. -H. Won, Y. -W. Jo, J. -H. Lee, M. Bawedin, S. Cristoloveanu, and J. -H. Lee, "High-performance GaN-based nanochannel FinFETs with/without AlGaN/GaN heterostructure," IEEE Trans. Electron Devices, vol. 60, no. 10, pp. 3012-3018, Oct. 2013. doi: 10.1109/TED.2013.2274660.

[34] J. Ma, C. Erine, P. Xiang, K. Cheng, and E. Matioli, "Multi-channel trigate normally-on/off AlGaN/GaN MOSHEMTs on Si substrate with high breakdown voltage and low ON-resistance," Appl. Phys. Lett., vol. 113, no. 242102 , pp. 1-5, Dec. 2018. doi: 10.1063/1.5064407.

(C) 2018 IEEE. Personal use of this material is permitted. Permission from IEEE must be obtained for all other uses, in any current or future media, including reprinting/republishing this material for advertising or promotional purposes, creating new collective works, for resale or redistribution to servers or lists, or reuse of any copyrighted component of this work in other works. 